\title{
Stochastic equation for the erosion of inclined topography
}

\author{
Romualdo Pastor-Satorras and Daniel H. Rothman \\ Department of Earth, Atmospheric, and Planetary Sciences \\ Massachusetts Institute of Technology, Cambridge, Massachusetts 02139
}

\begin{abstract}
We present a stochastic equation to model the erosion of topography with fixed inclination. The inclination causes the erosion to be anisotropic. A zero-order consequence of the anisotropy is the dependence of the prefactor of the surface height-height correlations on direction. The lowest higherorder contribution from the anisotropy is studied by applying the dynamic renormalization group. In this case, assuming an inhomogenous distribution of soil material, we find a one-loop estimate of the roughness exponents. The predicted exponents are in good agreement with new measurements made from seafloor topography.
\end{abstract}

PACS numbers: 92.40.Gc, 64.60.Ht, 05.60.+W, 64.60.Ak

The rich complexity of the Earth's surface, both on land and beneath the sea, is the result of physical mechanisms ranging from tectonic motion to surficial erosion [1,2]. Despite this variation, however, geologic surfaces show a certain degree of universality: they may often be characterized as self-affine 3, 3. over some range of length scales. This means that, if $h\left(\vec{x}, t_{0}\right)$ is the height of the surface at position $\vec{x}$ at some time $t_{0}$, then the "roughness", measured by the height-height static correlation function $C(\vec{x})=\left\langle\left(h\left(\vec{x}, t_{0}\right)-h\left(0, t_{0}\right)\right)^{2}\right\rangle^{1 / 2}$, grows as $x^{\alpha}$, where $\alpha$ is called the roughness exponent 国. Empirical measurements of $\alpha$ are numerous. While many indicate that $\alpha$ is small $(0.30<\alpha<0.55)$ [5.6], a number of other measurements show it to be large $(0.70<\alpha<0.85)$ [6]. Moreover, some measurements indicate that $\alpha$ crosses over from large to small values as length scales become greater than approximately $1 \mathrm{~km}[6]$. Motivated by these findings, we propose that the large values of $\alpha$ at small length scales may be explained by the influence of a preferred direction - downhill - for the flux of eroded material. We derive an anisotropic noisy diffusion equation to describe erosion at the small length scales where the preferred direction is fixed throughout space. Under the additional assumptions that the flux of eroded material increases with increasing distance downslope and that the dominant effects of noise are fixed in space, we find, using the dynamic renormalization group (DRG), a first-order estimate of the roughness exponents. New measurements of our own, made from the topography of the continental slope off the coast of Oregon, are in good agreement with our predictions. We find that our anisotropic theory significantly enriches previous isotropic continuum models [9,10] for two reasons. First, it predicts that correlations differ in different directions, and and second, it predicts that these correlations decay quantitatively differently than they do for isotropic topography.

Figure 1 i depicts the framework for our theory: a surface $h$ on a two-dimensional substrate. We refer to $h$ generically by the term landscape, and note that it inclination is fixed. The unit vector $\mathbf{e}_{h}$ is the "growth"

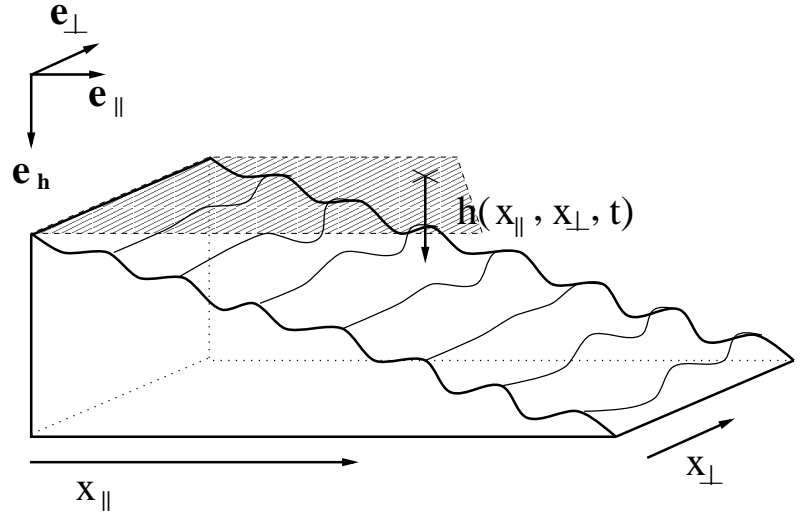

FIG. 1. Schematic configuration of an anisotropic landscape for the case $d=2$.

direction, which is measured downwards from the top of the slope. The preferred, downhill, direction is given by the unit vector $\mathbf{e}_{\|}$, while $\mathbf{e}_{\perp}$ represents a vector perpendicular to $\mathbf{e}_{\|}$and $\mathbf{e}_{h}$. Later, when applying the DRG, we will generalize to landscapes on a $d$-dimensional substrate; in this case $\mathbf{e}_{\perp}$ represents the subspace of all directions perpendicular to $\mathbf{e}_{\|}$and $\mathbf{e}_{h}$, and has dimension $d-1$. The configuration is completed by selecting fixed boundary conditions at the top of the slope, $x_{\|}=0$, or by imposing the symmetry $x_{\|} \rightarrow-x_{\|}$.

Due to the preferred direction $\mathbf{e}_{\|}$in Fig. 1, the statistical properties of $h$ may be anisotropic. Thus, if $h$ is self-affine, we expect different roughness exponents for correlations measured in each of the directions $\mathbf{e}_{\|}$and $\mathbf{e}_{\perp}$. Thus we define $\alpha_{\|}$and $\alpha_{\perp}$ such that $C_{\|}\left(x_{\|}\right) \sim x_{\|}^{\alpha_{\|}}$ for correlations along a fixed transect $\vec{x}_{\perp}^{0}=$ const., and $C_{\perp}\left(\vec{x}_{\perp}\right) \sim x_{\perp}^{\alpha_{\perp}}$ for correlations along a fixed transect $x_{\|}^{0}=$ const., where in general $\alpha_{\|} \neq \alpha_{\perp}$. These relations can be summarized in the single scaling form

$$
C\left(x_{\|}, \vec{x}_{\perp}\right) \sim b^{\alpha \|} C\left(b^{-1} x_{\|}, b^{-\zeta_{\|}} \vec{x}_{\perp}\right),
$$

where $\zeta_{\|}$is the anisotropy exponent. The exponents $\alpha_{\|}$ and $\alpha_{\perp}$ are related through $\alpha_{\perp}=\alpha_{\|} / \zeta_{\|}$. The exponent $\zeta_{\|}$accounts for the different rescaling factors along the two main directions. Since the space is anisotropic, when 
performing a scale change, we must rescale $x_{\|}$and $\vec{x}_{\perp}$ by different factors $b_{\|}$and $b_{\perp}$, respectively, if we are to recover a surface with the same statistical properties. We assume in our model that $\zeta_{\|}=\log b_{\perp} / \log b_{\|}=$const.

We seek a single stochastic equation for the landscape height $h$. Whereas others [9] have advocated the now classical, isotropic, non-conservative interface growth equation due to Kardar, Parisi, and Zhang (KPZ) [11], we assume here that the underlying soil is locally conserved such that

$$
\partial_{t} h=-\nabla \cdot \vec{J}+\eta
$$

where $\vec{J}$ is the current of soil per unit length. The soil however is not globally conserved, since it is lost at the bottom boundary. We also allow local conservation to be broken by the addition of a stochastic noise term $\eta$, discussed below.

Physically, the current $\vec{J}$ is expected to reflect two effects. First, we expect a local isotropic diffusing component, tending to smooth out the surface. Second, we expect an average global flow of dragged soil, directed mainly downhill. Thus we postulate the following form for the current:

$$
\vec{J}=-\nu \nabla h-\Gamma \nabla_{\|} h .
$$

The first term corresponds to Fick's law for diffusion, and represents the isotropic relaxational dynamics of the soil. The second term represents the average flow of soil that is dragged downhill, either due to the flow of water or the scouring of the surface by the flow of the soil itself. The direction of this term is given by the vector $\nabla_{\|} h \equiv \partial_{\|} h \mathbf{e}_{\|}$. The term $\Gamma$ plays the role of an anomalous anisotropic diffusivity. In order to gain insight into the role of $\Gamma$, consider the case in which erosion results from the stress exerted on the soil bed by an overland flow $q$ of water, where $q$ is the volumetric flow rate though unit area perpendicular to the direction of steepest descent. The greater $q$ is, the stronger is the stress [1, 2]. Moreover, since $q$ flows downhill, it increases with distance downslope. Thus $\Gamma$ must be an increasing function of $x_{\|}$. Since the fixed inclination implies that $h$ increases with $x_{\|}$, we choose to parameterize the anomalous diffusion as a function of the height such that $\Gamma \equiv \Gamma(h)$ [12]. Defining $\Gamma(h)=\lambda_{0}+g(h)$, with $g(0)=0$ and $G(h)=\int g(h) d h$, we substitute Eq. (3) into (2). Since $g(h) \partial_{\|} h=[d G(h) / d h] \partial_{\|} h=\partial_{\|} G(h)$, where we have used the chain rule for the second equality, we obtain

$$
\partial_{t} h=\nu_{\|} \partial_{\|}^{2} h+\nu_{\perp} \nabla_{\perp}^{2} h+\partial_{\|}^{2} G(h)+\eta,
$$

where $\nu_{\perp}=\nu$ and $\nu_{\|}=\nu+\lambda_{0}$.

Even in the absence on any nonlinearity, fundamental conclusions may be drawn from (4). By setting $g=0$ (i.e., by considering $\Gamma(h)=\lambda_{0} \equiv$ const.), we obtain a linear equation which is an anisotropic counterpart of the
Edwards-Wilkinson equation [4]. In can then be easily shown [ 1 that the correlation functions along the main directions $\mathbf{e}_{\|}$and $\mathbf{e}_{\perp}$ are inversely proportional to the square root of the diffusivities $\nu_{\|}$and $\nu_{\perp}$ respectively, that is, $C_{\perp} / C_{\|} \sim\left(\nu_{\|} / \nu_{\perp}\right)^{1 / 2}$. In other words, since the preferred direction gives $\nu_{\|}>\nu_{\perp}$, the topography is quantitatively rougher, at all scales and by the same factor, in the perpendicular direction than in the parallel direction.

In order to obtain more information on the scaling properties of Eq. (雨), we have studied it using the DRG. Assuming that $\Gamma(h)$ is an analytical function, we can perform a Taylor expansion in powers of $h$. Since all odd powers of $h$ must vanish in order to the preserve the joint symmetry $h \rightarrow-h, \vec{J} \rightarrow-\vec{J}$ in Eq. (2), we are left at lowest order with $g(h) \simeq \lambda_{2} h^{2}$. By dimensional analysis one can check that all the terms in this expansion are relevant under rescaling. However, the flux $Q\left(x_{\|}\right)$of the erosive agent (water or soil) flowing on the surface should grow no faster than $Q\left(x_{\|}\right) \sim x_{\|}^{d}$. Then, taking $h \sim x_{\|}$, we find that the terms in $g(h)$ should be of order $h^{d}$ or less. Specializing to the case of $d=2$ (i.e., real surfaces), we then find it reasonable to truncate $g$ at second order, such that Eq. (14) takes the form

$$
\partial_{t} h=\nu_{\|} \partial_{\|}^{2} h+\nu_{\perp} \nabla_{\perp}^{2} h+\frac{\lambda}{3} \partial_{\|}^{2}\left(h^{3}\right)+\eta,
$$

where $\lambda=\lambda_{2}$. Note that Eq. (5) differs from the anisotropic driven diffusion equation of Hwa and Kardar 113. because the form of our current $\vec{J}$ is suggested not only by symmetry arguments, but also by the physics of erosion.

We now address the issue of noise. We distinguish two different sources. First, we may allow a term of "annealed" noise, $\eta_{t}(\vec{x}, t)$, depending on time and position, and describing a random, external forcing, due to, for example, inhomogeneous rainfall. We assume that this noise is isotropic, Gaussian distributed, with zero mean, and uncorrelated such that $\left\langle\eta_{t}(\vec{x}, t) \eta_{t}\left(\vec{x}^{\prime}, t^{\prime}\right)\right\rangle=$ $2 D_{t} \delta^{(d)}\left(\vec{x}-\vec{x}^{\prime}\right) \delta\left(t-t^{\prime}\right)$. Second, we may have a term of "quenched" noise to account for the heterogeneity of the soil, mimicking the variations in the erodibility of the landscape 8 . We represent this randomness by a source of Gaussian static noise $\eta_{s}(\vec{x})$, with correlations $\left\langle\eta_{s}(\vec{x}) \eta_{s}\left(\vec{x}^{\prime}\right)\right\rangle=2 D_{s} \delta^{(d)}\left(\vec{x}-\vec{x}^{\prime}\right)$. This form of noise has been previously proposed to model soil heterogeneity in cellular automata models of fluvial networks [14]. In the following we consider the limits (i) $\eta_{s}=0\left(D_{s} \ll D_{t}\right)$, corresponding to a situation of random external forcing and homogeneous composition of soil, and (ii) $\eta_{t}=0$ $\left(D_{s} \gg D_{t}\right)$, representing the limit in which the external forcing is constant and the most essential source of noise is the inhomogeneous composition of the soil.

Application of the DRG follows the procedure used in Refs. 13, 15]. In Fourier space we proceed by integrating 
over the shell of large wave vectors $\Lambda e^{-l}<k<\Lambda$, where $\Lambda$ is the wave vector upper cutoff and $e^{l}$ is the rescaling factor, and by subsequently rescaling the system back to its original size through the transformation $\vec{x}_{\perp} \rightarrow e^{l} \vec{x}_{\perp}$, $x_{\|} \rightarrow e^{l \zeta_{\perp}} x_{\|}, h \rightarrow e^{l \alpha_{\perp}} h$, and $t \rightarrow e^{l z_{\perp}} t$. The anisotropy is explicitly included in the exponent $\zeta_{\perp} \equiv \zeta_{\|}^{-1}$. To lowest order in perturbation theory, both limits (i) and (ii) above provide the same form for the renormalization group flow equations:

$$
\begin{aligned}
& \frac{d \nu_{\|}}{d l}=\nu_{\|}\left(z_{\perp}-2 \zeta_{\perp}+\bar{\lambda}_{i}\right), \quad \frac{d \nu_{\perp}}{d l}=\nu_{\perp}\left(z_{\perp}-2\right) \\
& \frac{d \lambda}{d l}=\lambda\left(z_{\perp}+2 \alpha_{\perp}-2 \zeta_{\perp}-\frac{3}{2} \bar{\lambda}_{i}\right) \\
& \frac{d D_{i}}{d l}=D_{i}\left(\kappa_{i} z_{\perp}-2 \alpha_{\perp}-\zeta_{\perp}-d+1\right),
\end{aligned}
$$

where $i=t, s$ stands for the limits (i) and (ii) above, respectively. Here $\bar{\lambda}_{i}$ is an effective coupling constant, depending on the type of noise: $\bar{\lambda}_{t}=\lambda D_{t} K_{d-1} \Lambda^{d-2} / 2 \nu_{\|}^{3 / 2} \nu_{\perp}^{1 / 2}$ in (i), and $\bar{\lambda}_{s}=$ $\lambda D_{s} K_{d-1} \Lambda^{d-4} / 2 \nu_{\|}^{3 / 2} \nu_{\perp}^{3 / 2}$ in (ii), with $K_{d}=S_{d} /(2 \pi)^{d}$ and $S_{d}$ the surface area of a $d$-dimensional unit sphere. The value of the correction factor $\kappa_{i}$ is $\kappa_{t}=1$ and $\kappa_{s}=2$. The flow equations for $\nu_{\perp}$ and $D_{i}$ are exact to all orders in the perturbation expansion $[13,16]$. They provide us with the exact result $z_{\perp}=2$ [17]. The effective coupling flows under rescaling as

$$
\frac{d \bar{\lambda}_{i}}{d l}=\bar{\lambda}_{i}\left(\varepsilon_{i}-3 \bar{\lambda}_{i}\right)
$$

where $\varepsilon_{i}=d_{c}^{(i)}-d$, and $d_{c}^{(i)}$ is the critical dimension for each particular limit, $d_{c}^{(t)}=2$ and $d_{c}^{(s)}=4$. The stable fixed points of (6) are $\bar{\lambda}_{i}^{*}=0$ for $d>d_{c}^{(i)}$ and $\bar{\lambda}_{i}^{*}=\varepsilon_{i} / 3$ for $d<d_{c}^{(i)}$. For $d>d_{c}^{(i)}$ the critical exponents attain in both limits their mean-field values $\alpha_{\perp}^{M F}=0$, $\zeta_{\perp}^{M F}=1$, and $z_{\perp}^{M F}=2$. On the other hand, for $d<d_{c}^{(i)}$, the critical exponents computed at first order in the $\varepsilon$ expansion are:

$$
\alpha_{\perp(i)}=\frac{5 \varepsilon_{i}}{12}, \quad \zeta_{\perp(i)}=1+\frac{\varepsilon_{i}}{6} .
$$

The physically relevant dimension for erosion is $d=2$. In the limit of thermal noise this corresponds to the critical dimension. By continuity, the exponents are $\alpha_{\perp}=\alpha_{\|}=0$ and $\zeta_{\perp}=\zeta_{\|}=1$. This result is consistent with a flat landscape, with logarithmic corrections to the roughness 㕶. However, we still expect anisotropy to appear in the prefactor of the correlation functions $C_{\|}$ and $C_{\perp}$, as argued above. On the other hand, in the limit of static noise we are below the critical dimension, and (7) is applicable. Substituting $\varepsilon_{s}=2$ we obtain the roughness exponents

$$
\alpha_{\perp}=\frac{5}{6} \simeq 0.83, \quad \alpha_{\|}=\frac{\alpha_{\perp}}{\zeta_{\perp}}=\frac{5}{8} \simeq 0.63 .
$$

The values (8) predicted for $\alpha_{\perp}$ and $\alpha_{\|}$are in reasonable agreement with previous measures made at small length scales [6, [7]. However, these measurements were either averaged over all directions or the direction of the measurements was not reported. Thus, to check our results with a natural landscape that has an unambiguous preferred direction, we have analyzed digital bathymetric maps of the continental slope off the coast of Oregon. In this case the slope results from the relatively abrupt increase in the depth of the seafloor as the continental shelf gives way to the deeper continental rise. Figure $2 \mathrm{a}$ shows one portion of this region. Here the main feature of the topography is a deep incision called a submarine canyon. In this region, submarine canyons are thought to have resulted from seepage-induced slope failure [18], which occurs when excess pore pressure within the material overcomes the gravitational and friction forces on the surface of the material, causing the slope to become unstable. Slope instabilities then create submarine avalanches, which themselves can erode the slope as they slide downwards.

Figure $2 \mathrm{~b}$ shows the height-height correlation functions $C_{\|}$and $C_{\perp}$, corresponding, respectively, to the parallel and perpendicular directions of the seafloor topography in Fig. 2a. The computation of $C_{\perp}$ follows from its definition but the computation of $C_{\|}$requires some comment. The fluctuations measured by $C_{\|}$must be defined with respect to an appropriate average profile. Briefly, one expects that geologic processes other than erosion (e.g., tectonic stresses) are responsible for long-wavelength deformation in the parallel direction. We may estimate such systematic corrections by computing the mean profile in the parallel direction: $h_{\mathrm{av}}\left(x_{\|}\right)=L_{\perp}^{-1} \int d x_{\perp} h\left(x_{\|}, x_{\perp}\right)$. We then compute $C_{\|}$from the fluctuations of the detrended surface $\tilde{h}=h-h_{\text {av }}\left(x_{\|}\right)$. ¿From both $C_{\|}$and $C_{\perp}$ we find that the least-squares estimates of the roughness exponents, $\alpha_{\|} \simeq 0.67$ and $\alpha_{\perp} \simeq 0.78$, exhibit a surprisingly good fit to our theoretical predictions (8).

We have also measured $C_{\|}$and $C_{\perp}$ in some desert environments. In these cases (not shown), we did not obtain conclusive power law scaling, but we always found $C_{\perp} / C_{\|}>1$, as predicted by the linear theory. Thus, while the example of Figure 2 may be in some sense specialized, one of our main predictions - that the topography in the perpendicular direction is rougher than the topography in the parallel direction - seems to be of fairly general validity.

In conclusion, we note that the main elements of our theory are the conservation of the eroded material, randomness of either the landscape or the forcing, and the presence of a preferred direction for the material transport. The latter assumption leads to an anisotropic equation that applies, in principle, to any erosive process with the appropriate lack of symmetry. In the usual geological setting, however, the anisotropy applies specifically to a 

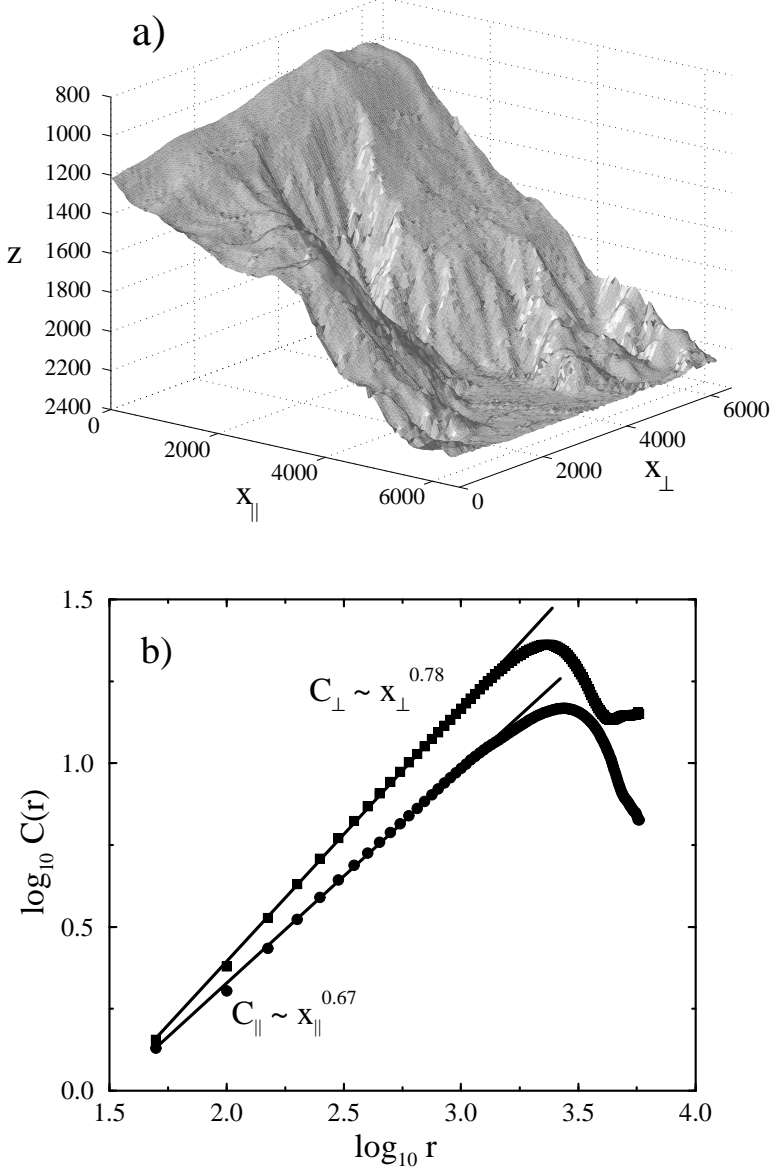

FIG. 2. (a) Digital map of a submarine canyon off the coast of Oregon, located at coordinates $44^{\circ} 40^{\prime} \mathrm{N}, 125^{\circ} 45^{\prime}$ $\mathrm{W}$. The vertical axis represents depth below sea level. Distances are measured in meters. (b) Height-height correlation functions computed along the parallel $\left(C_{\|}\right)$and perpendicular $\left(C_{\perp}\right)$ directions. Solid lines are least-squares fits to the scaling region.

surface of fixed inclination which, in turn, implies that our theory should only apply locally, to the relatively small scales where the preferred direction of transport is approximately constant. Because the anisotropy should vanish at large length scales, these large scale features should be presumably described by an isotropic theory, such as the KPZ equation 19,11. Indeed, the KPZ equation predicts exponents that are approximately consistent with large scale observations. Since these predictions differ from ours, it may be possible to use our results to distinguish statistically between features of the landscape due to erosion and features due to larger-scale processes, such as tectonic deformation.

R.P.S. acknowledges financial support from the Ministerio de Educación y Cultura (Spain). The work of D.H.R. was supported in part by NSF grant EAR-
[1] A. E. Scheidegger, Theoretical Geomorphology, 2 ed. (Springer-Verlag, Berlin, 1991).

[2] I. Rodriguez-Iturbe and A. Rinaldo, Fractal River Basins: Chance and Self-Organization (Cambridge University Press, Cambridge, 1997).

[3] B. B. Mandelbrot, The Fractal Geometry of Nature (Freeman, San Francisco, 1982).

[4] A.-L. Barabási and H. E. Stanley, Fractal Concepts in Surface Growth (Cambridge University Press, New York, 1995).

[5] W. I. Newman and D. L. Turcotte, Geophys. J. Int. 100, 433 (1990); D. L. Turcotte, Fractals and Chaos in Geology and Geophysics (Cambridge University Press, New York, 1992).

[6] D. M. Mark and P. B. Aronson, Math. Geol. 16, 671 (1984); M. Matsushita and S. Ouchi, Physica D 38, 246 (1989); S. Ouchi and M. Matsushita, Geomorphology 5, 15 (1992); C. G. Chase, Geomorphology 5, 39 (1992); N. A. Lifton and C. G. Chase, Geomorphology 5, 77 (1992).

[7] G. I. Barenblatt, A. V. Zhivago, Yu. P. Neprochnov, and A. A. Ostrovskiy, Oceanology 24, 695 (1984); L. E. Gilbert, PAGEOPH. 131, 241 (1989); D. Norton and S. Sorenson, PAGEOPH. 131, 77 (1989).

[8] A. Czirók, E. Somfai, and J. Vicsek, Phys. Rev. Lett. 71, 2154 (1993).

[9] D. Sornette and Y.-C. Zhang, Geophys. J. Int. 113, 382 (1993).

[10] A. Giacometti, A. Maritan, and J. R. Banavar, Phys.Rev. Lett. 75, 577 (1995); K. Sinclair and R. C. Ball, Phys. Rev. Lett. 76, 3360 (1996); E. Somfai and L. M. Sander, Phys. Rev. E 56, R5 (1997); J. R. Banavar et al., Phys.Rev. Lett. 78, 4522 (1997).

[11] M. Kardar, G. Parisi, and Y.-C. Zhang, Phys. Rev. Lett. 56, 889 (1986); E. Medina, T. Hwa, M. Kardar, and Y.C. Zhang, Phys. Rev. A 39, 3053 (1989).

[12] Arguments supporting this parametrization are also given by J. D. Pelletier, (preprint physics/9705033) (1997).

[13] T. Hwa and M. Kardar, Phys. Rev. A 45, 7002 (1992).

[14] G. Caldarelli et al., Phys. Rev. E 55, R4865 (1997).

[15] A. Corral and A. D. Guilera, Phys. Rev. E 55, 2434 (1997).

[16] Z.-W. Lai and S. D. Sarma, Phys. Rev. Lett. 66, 2348 (1991).

[17] The dynamic critical exponent $z$ measures the saturation length of correlations as a function of time. However, since the time scales for geomorphologic evolution are many orders of magnitude larger than those possibly available for observation, speculations about the actual value of $z$ are meaningless.

[18] D. L. Orange, R. S. Anderson, and N. A. Breen, GSA Today 4, 29 (1994). 